\title{
Remission of Chronic Acquired Demyelinating Polyneuropathy Associated With Paraproteinemia After Removal of Skull Plasmacytoma
}

\author{
Sung-Yeon Sohnª, bin-Ok Kima, Soo Joo Lee ${ }^{\mathrm{a}}$
}

\begin{abstract}
Chronic acquired demyelinating polyneuropathy can be caused by various etiologies. Although chronic inflammatory demyelinating polyradiculoneuropathy (CIDP) is the most common chronic acquired demyelinating polyneuropathy, currently there is no suitable biomarker to diagnose CIDP; hence misdiagnosis of CIDP has not been unusual. Herein, we present a patient with initially presumed CIDP, who maintains disease remission after early surgical removal of solitary plasmacytoma during 1-year follow-up. A 55-year-old woman was referred to our hospital due to progressive upper and lower extremity weakness from 6 months ago. Nerve conduction study findings were compatible with acquired demyelinating polyneuropathy, fulfilling electrodiagnostic criteria of CIDP. Serum electrophoresis suggested monoclonal gammopathy of lambda type, but the bone marrow biopsy showed no evidence of neoplastic plasma cell infiltration. Brain magnetic resonance imaging revealed an osteolytic mass involving the right parietal skull and adjacent dura mater. Total excision of the skull mass was performed. The biopsy result was compatible with plasmacytoma. Even without long term administration of high dose corticosteroid, the patient not only has continued to improve clinically, but also showed markedly improved electrophysiological findings during 1-year follow-up.
\end{abstract}

Keywords: Chronic acquired demyelinating polyneuropathy; Chronic inflammatory demyelinating polyradiculoneuropathy; Paraproteinemia; Solitary plasmacytoma

\section{Introduction}

Chronic acquired demyelinating polyneuropathy mainly includes chronic inflammatory demyelinating polyradiculoneuropathy (CIDP) and its variants, multifocal motor neuropathy

Manuscript submitted November 19, 2017, accepted December 6, 2017

aDepartment of Neurology, Eulji University Hospital, Daejeon, Korea ${ }^{b}$ Corresponding Author: Sung-Yeon Sohn, Department of Neurology, Eulji University Hospital, 95 Dunsanseo-ro, Seo-gu, Daejeon, Korea.

Email: sungyeonsohn@gmail.com

doi: https://doi.org/10.14740/jnr462w
$(\mathrm{MMN})$, paraproteinemic demyelinating neuropathy (PDN) including neuropathy associated anti-myelin associated glycoprotein (MAG) antibodies and polyneuropathy, organomegaly, endocrinopathy/edema, M-protein and skin changes (POEMS) syndrome [1]. The most common chronic acquired demyelinating polyneuropathy is CIDP, with a prevalence of 1 to 9 cases per 100,000 [2]. Since currently there is no suitable biomarker to diagnose CIDP, patients presenting as chronic acquired demyelinating polyneuropathy are sometimes misdiagnosed as CIDP. One study reported that $18 \%$ of the patients included in a clinical trial for CIDP were not in fact CIDP, and they were diagnosed correctly only after the failure to initial standard treatment for CIDP $[1,3]$.

We present herein a patient who was initially diagnosed as CIDP, but was treated successfully by performing early excision of skull plasmacytoma. The patient maintains disease remission state after surgical removal of plasmacytoma during 1-year follow-up, without long term administration of high dose corticosteroid.

\section{Case Report}

A 55-year-old woman was referred from local orthopedic clinic due to progressive upper and lower extremity weakness. Her symptoms began insidiously from 6 months before visiting our hospital, slowly progressed from distal to proximal muscles. She was unable to walk without assistance from 1 month before admission. Previous medical history was unremarkable except diabetes mellitus. Electrocardiogram revealed atrial fibrillation. On neurological examination, there was weakness in the arms proximally (grade 4 on the Medical Research Council scale, where grade 5 is normal) and distally (grade 4-). The strength of the legs was grade 4- proximally and grade 2 distally. The sensation of all modalities was diminished especially in the lower extremities. Fasciculation was visible on her left arm.

Laboratory study revealed a normal complete blood count, serum creatinine, erythrocyte sedimentation rate, and mild hypothyroidism (free T4 $0.85 \mathrm{ng} / \mathrm{dL}$ (reference range: 0.89 - 1.76 $\mathrm{ng} / \mathrm{dL}$ ), thyroid-stimulating hormone $6.28 \mu \mathrm{IU} / \mathrm{L}$ (reference range: 0.55 - $4.78 \mu \mathrm{IU} / \mathrm{L}$ ), T3 $35 \mathrm{ng} / \mathrm{dL}$ (reference range: 65 $150 \mathrm{ng} / \mathrm{dL})$ ). Serum rheumatoid factor, anti-nuclear antibody, anti-neutrophil cytoplasmic antibody, anti-ganglioside antibody including anti-MAG antibody were not detected. Serum 

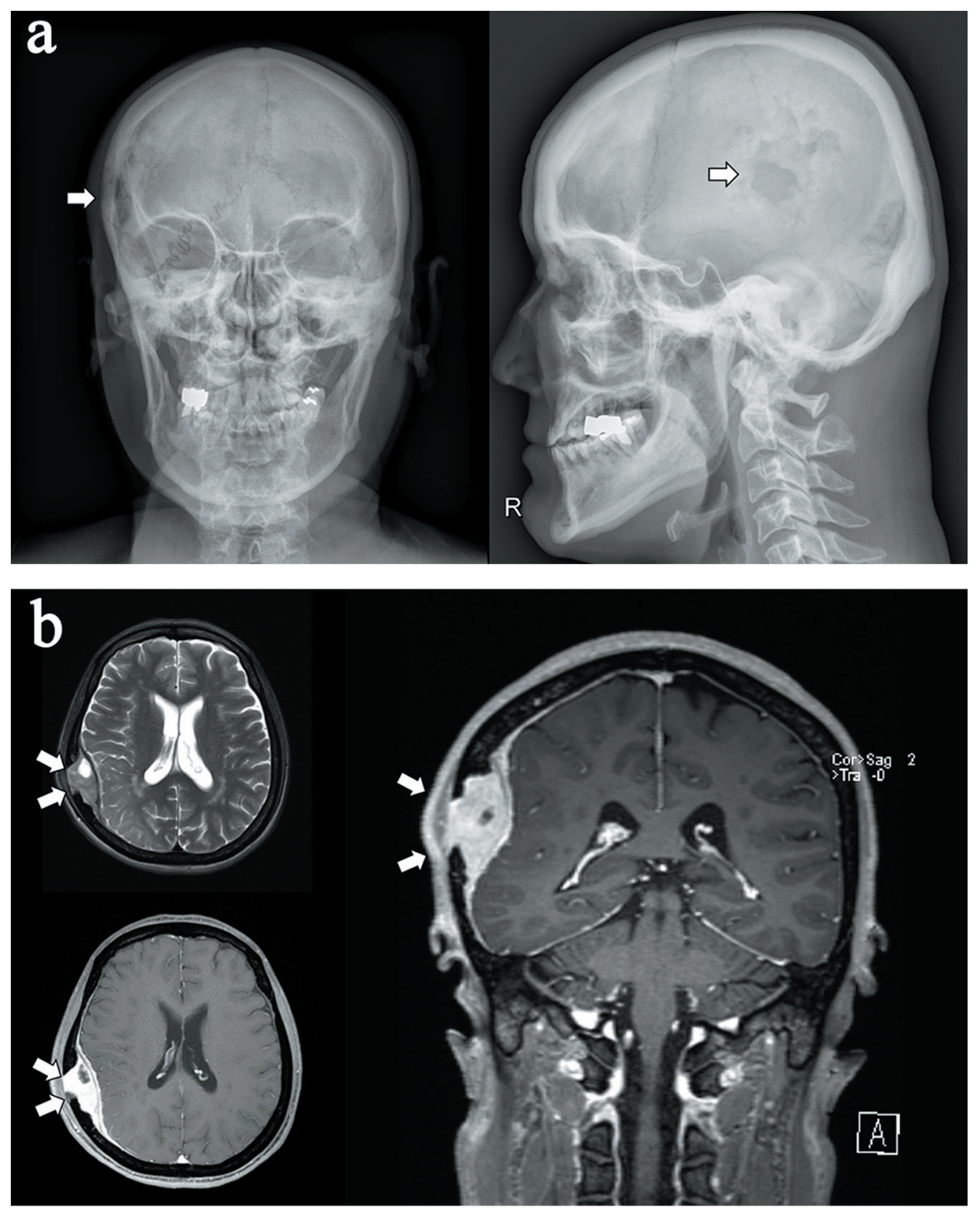

Figure 1. (a) Skull X-ray of the patient which revealed an osteolytic mass in the right parietal skull (arrows). (b) Brain magnetic resonance imaging of the patient which revealed an intermediate T2 signal intensity, well enhancing osteolytic mass (about $53 \times$ $20 \times 63 \mathrm{~mm}$ ) involving the right parietal skull, right parieto-temporal epidural space and adjacent dura (arrows).

protein electrophoresis showed increased gamma portion, and serum immunofixation electrophoresis suggested monoclonal gammopathy of lambda type. Urine protein electrophoresis revealed Bence Jones protein monomer. Nerve conduction study revealed motor conduction blocks in left median and bilateral ulnar nerves, as well as marked slowing of motor conduction velocity in bilateral median and ulnar nerves. Sensory nerve conduction velocity and sensory nerve action potential of bilateral median and ulnar nerves were decreased. Electrical stimulation of both motor and sensory nerves of lower extremities showed no response. The results of nerve conduction study suggested CIDP, fulfilling the electrodiagnostic criteria proposed by European Federation Neurological Society [4]. Cerebrospinal fluid analysis showed increased protein level (219.5 mg/dL, reference range: 18 - $40 \mathrm{mg} / \mathrm{dL}$ ) without pleocytosis.
Bone marrow biopsy showed moderate granulocytic hyperplasia without evidence of neoplastic plasma cell infiltration, suggestive of monoclonal gammopathy of undetermined significance (MGUS). Under the initial diagnosis of CIDP with MGUS, intravenous methylprednisolone $1 \mathrm{~g}$ /day was started, along with subsequent diagnostic procedures. Whole body skeletal X-ray revealed old multiple rib fractures with callus formation in the right ribs, old fracture in the right clavicle, and a focal osteolytic lesion in the right parietal bone (Fig. 1a). Brain magnetic resonance imaging (MRI) revealed a well enhancing osteolytic mass involving the right parietal skull and adjacent dura mater (Fig. 1b).

Corticosteroid was fully tapered within 2 weeks. Elective surgical removal of the skull mass was performed. The obtained biopsy result was compatible with plasmacytoma. Immunohistochemistry showed CD138 positive, CD56 focal 

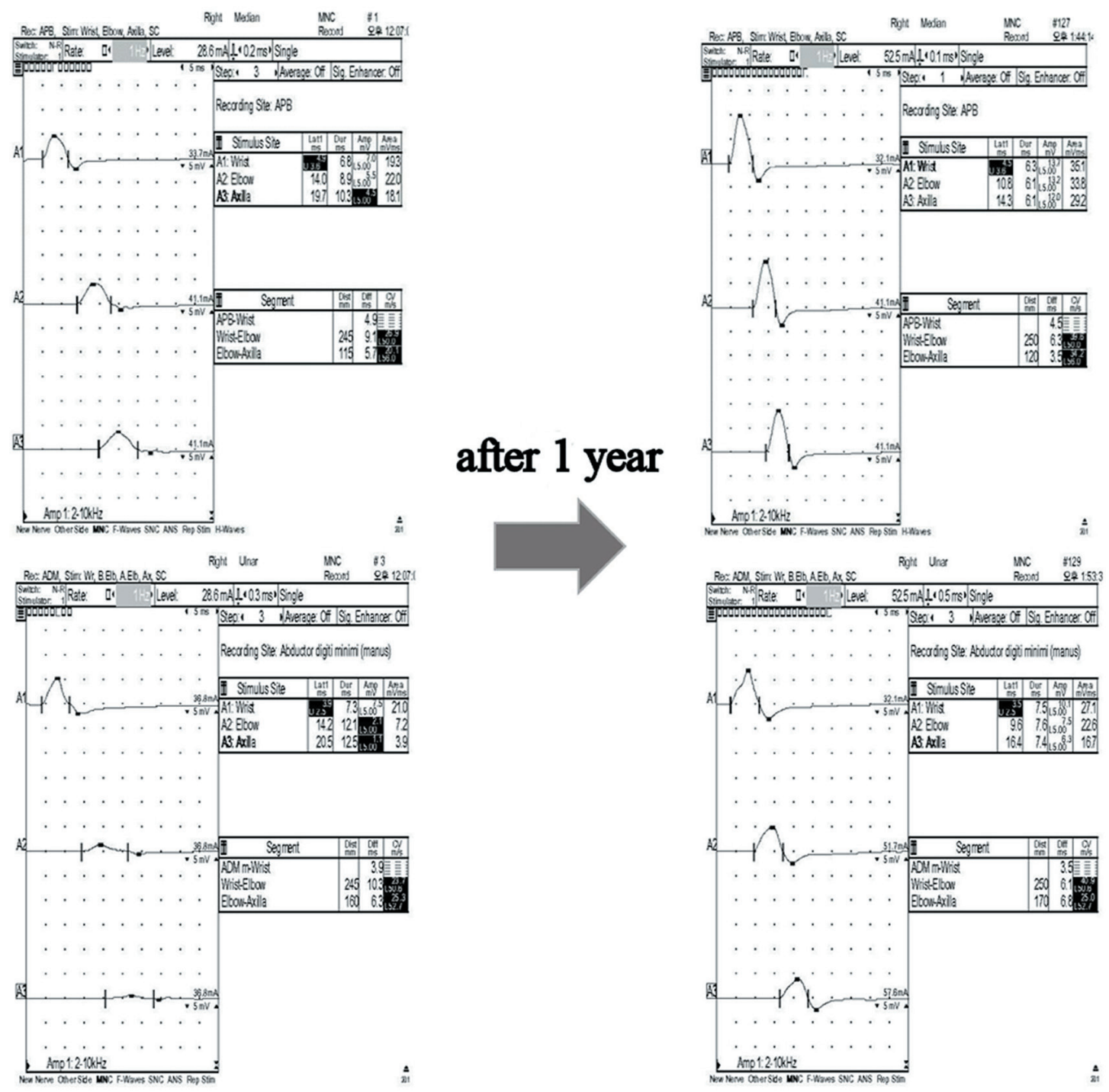

Figure 2. Nerve conduction study of the patient. Note the improvement of conduction blocks in median and ulnar nerves 1 year after removal of skull plasmacytoma.

positive, epithelial membrane antigen (EMA) positive, plasma cell proliferation and lambda light chain restriction. One year after surgery, the patient not only has continued to improve clinically, but also showed markedly improved electrophysiological findings (Fig. 2).

\section{Discussion}

The close association between CIDP and paraproteinemia has been well recognized $[5,6]$. Recently, CIDP associated with paraproteinemia is viewed as a distinct entity, since the clinical course and management strategies vary $[1,7,8]$. Paraproteinemic demyelinating neuropathy (PDN) may be an appropriate term, despite there is no consensus as to whether it should be considered the same as CIDP [9]. CIDP-like presentation in PDN is not uncommon, including neuropathy associated with MGUS (IgM, IgG or IgA), anti-MAG antibody, Waldenstrom's macroglobulinemia, Chronic Ataxic Neuropathy with Ophthalmoplegia, IgM Monoclonal gammopathy, cold Agglutinins and Disialoganglioside (IgM anti-GD1b/GQ1b) antibodies (CANOMAD), POEMS syndrome, and rarely immunoglobulin light chain (AL) amyloidosis, cryoglobulinemia, lytic multiple myeloma, and neurolymphomatosis $[10,11]$.

Our patient presented with progressive symmetric weakness and sensory disturbance in both proximal and distal muscles, indistinguishable from symptoms of typical CIDP. However, she showed marked improvement after removal of skull plasmacytoma without long term maintenance of corticosteroid, making the clinical diagnosis of typical CIDP unlikely.

Solitary plasmacytoma presenting as chronic demyelinating polyneuropathy is a rare condition, hence the clinical consequence is largely unknown. The majority of literature reported an improvement of polyneuropathy after management 
of plasmacytoma [5, 12-14], whereas some authors reported development of POEMS syndrome despite initial management of solitary plasmacytoma after several years $[15,16]$. Although our patient has been maintaining remission state during 1-year follow-up period, close observation may be needed, considering possibility of evolvement to POEMS or multiple myeloma.

Treatment of chronic demyelinating polyneuropathy should be tailored according to underlying etiology. Chronic acquired demyelinating polyneuropathy associated with skull plasmacytoma may be successfully managed with surgical removal of the causative lesion.

\section{Conflict of Interest}

The authors do not have any conflict of interest to declare.

\section{References}

1. Latov N. Diagnosis and treatment of chronic acquired demyelinating polyneuropathies. Nat Rev Neurol. 2014;10(8):435-446.

2. Mathey EK, Park SB, Hughes RA, Pollard JD, Armati PJ, Barnett $\mathrm{MH}$, Taylor BV, et al. Chronic inflammatory demyelinating polyradiculoneuropathy: from pathology to phenotype. J Neurol Neurosurg Psychiatry. 2015;86(9):973-985.

3. Eftimov F, Vermeulen M, van Doorn PA, Brusse E, van Schaik IN. Long-term remission of CIDP after pulsed dexamethasone or short-term prednisolone treatment. Neurology. 2012;78(14):1079-1084.

4. Joint Task Force of the E, the PNS. European Federation of Neurological Societies/Peripheral Nerve Society Guideline on management of chronic inflammatory demyelinating polyradiculoneuropathy: report of a joint task force of the European Federation of Neurological Societies and the Peripheral Nerve Society--First Revision. J Peripher Nerv Syst. 2010;15(1):1-9.

5. Koo YS, Kim BJ. A patient with chronic inflammatory demyelinating polyneuropathy associated with an intracranial plasmacytoma. Am J Phys Med Rehabil.
2010;89(2):173-174.

6. Koller H, Kieseier BC, Jander S, Hartung HP. Chronic inflammatory demyelinating polyneuropathy. N Engl J Med. 2005;352(13):1343-1356.

7. Nobile-Orazio E, Bianco M, Nozza A. Advances in the treatment of paraproteinemic neuropathy. Curr Treat Options Neurol. 2017;19(12):43.

8. Rinaldi S, Bennett DL. Pathogenic mechanisms in inflammatory and paraproteinaemic peripheral neuropathies. Curr Opin Neurol. 2014;27(5):541-551.

9. Hughes RA, Brainin M, Gilhus NE. European handbook of neurological management. John Wiley \& Sons, 2nd edition. 2008.

10. Mauermann ML. Neurologic Complications of Lymphoma, Leukemia, and Paraproteinemias. Continuum (Minneap Minn). 2017;23(3, Neurology of Systemic Disease):669-690.

11. Joint Task Force of the EFNS and the PNS. European Federation of Neurological Societies/Peripheral Nerve Society Guideline on management of paraproteinemic demyelinating neuropathies. Report of a Joint Task Force of the European Federation of Neurological Societies and the Peripheral Nerve Society--first revision. J Peripher Nerv Syst. 2010;15(3):185-195.

12. Mankodi AK, Rao CV, Katrak SM. Solitary plasmacytoma presenting as peripheral neuropathy: a case report. Neurol India. 1999;47(3):234-237.

13. Nakano A, Mitsui T, Endo I, Takeda Y, Ozaki S, Matsumoto T. Solitary plasmacytoma with VEGF overproduction: report of a patient with polyneuropathy. Neurology. 2001;56(6):818-819.

14. Velasco R, Bau L, Povedano M, Petit J, Lucas A, Bruna J. Sensory-motor polyradiculoneuropathy as the first manifestation of sternum bone plasmacytoma only revealed by bone scintigraphy. Neuromuscul Disord. 2009;19(1):5961.

15. Shindo M, Sato K, Yamamoto M, Toki Y, Hatayama M, Ito S, Ichiki K, et al. Development of POEMS syndrome after an initial manifestation of solitary plasmacytoma. Int J Hematol. 2011;93(6):815-821.

16. Rathore NK, Sharma A, Sathpathy SP, Walke R. Oncological progression of bone plasmacytoma to POEMS syndrome. J Cancer Res Ther. 2011;7(3):366-367. 